\title{
Anisotropy of Spin Relaxation in Metals
}

\author{
Bernd Zimmermann, Phivos Mavropoulos, ${ }^{*}$ Swantje Heers, Nguyen H. Long, Stefan Blügel, and Yuriy Mokrousov \\ Peter Grünberg Institut and Institute for Advanced Simulation, Forschungszentrum Jülich and JARA, 52425 Jülich, Germany
} (Received 9 May 2012; published 5 December 2012)

\begin{abstract}
The concept of anisotropy of spin relaxation in nonmagnetic metals with respect to the spin direction of the injected electrons relative to the crystal orientation is introduced. The effect is related to an anisotropy of the Elliott-Yafet parameter, arising from a modulation of the decomposition of the spin-orbit Hamiltonian into spin-conserving and spin-flip terms as the spin quantization axis is varied. This anisotropy, reaching gigantic values for uniaxial transition metals (e.g., 830\% for hcp Hf) as densityfunctional calculations show, is related to extended "spin-flip hot areas" on the Fermi surface created by the proximity of extended sheets of the surface, or "spin-flip hot loops" at the Brillouin zone boundary, and has no theoretical upper limit. Possible ways of measuring the effect as well as consequences in application are briefly outlined.
\end{abstract}

DOI: 10.1103/PhysRevLett.109.236603

PACS numbers: 72.25.Rb, 72.25.Ba, 76.30.Pk, 75.76.+j

Spin-relaxation processes are of fundamental importance for the realization of spintronic devices, which aim at utilizing the electron spin degree of freedom for processing and transfer of information [1]. A nonequilibrium spin distribution will equilibrate, and the corresponding information will be lost on a time scale of the spin-relaxation time $T_{1}$. The dominant spin-relaxation mechanism in structure- and bulk-inversion symmetric, nonmagnetic metals is the Elliott-Yafet mechanism [2,3], which is due to scattering of electrons at phonons or impurities. Owing to the presence of spin-orbit coupling (SOC) in a solid, such scattering events will flip the spin of an electron with a certain probability, which depends on both the wave functions of the ideal crystal and the scattering potential. Within the Elliott approximation in the diffusive regime, one obtains that the ratio between the momentum and spinrelaxation times is proportional to the Elliott-Yafet (or spin-mixing) parameter $b^{2}$, which is a property of the ideal crystal only [2,3]. While $b^{2}$ has long since been accepted as a measure of spin relaxation, the anisotropy of $b^{2}$ has not been analyzed on a theoretical basis so far [4].

In an experiment measuring spin relaxation, the injected electrons are characterized by the axis of spin polarization which is determined, e.g., by the external magnetic field in conduction-electron spin resonance or by the direction of magnetization of ferromagnetic leads in spin-injection or giant-magnetoresistance experiments. For our purposes we call this direction the spin quantization axis (SQA) $\hat{\mathbf{s}}$. As it turns out, the SQA relative to the crystal lattice matters for the value of $b^{2}$, and not just by a little. This gives us the notion of anisotropy of the Elliott-Yafet parameter and consequently of spin relaxation. It will thus make a difference in an experiment if spins are injected into a metal from a ferromagnet whose magnetization is normal to the interface, compared to being parallel, as long as the metal exhibits a preferential crystalline orientation. Since the spin population decays exponentially with respect to the distance from the injection point, with $T_{1}$ entering in the exponent, the anisotropy can make a difference between, e.g., a well-operating and a defective giant-magnetoresistance junction. One realizes also that we are faced with an anisotropy of an irreversible process, i.e., of the entropy production during relaxation. This can have far-reaching consequences, for example, in the spin-entropy-induced Peltier cooling in nanojunctions [7]. In this respect this anisotropy is fundamentally different from other, known SOC effects, such as the magnetocrystalline anisotropy energy of ferromagnets or the anisotropic magnetoresistance which arise from small changes of the band energies depending on the magnetization direction. In a nonmagnetic metal, the choice of the SQA does not influence the band energies, but it manifests itself through matrix elements determining the orbital and spin character of the Bloch states, which can have a profound influence on spin transport [8]. One also expects an anisotropy in the spin susceptibility, since the spin-mixing parameter of a state is directly related to its response to a Zeeman field.

In this Letter, we investigate the anisotropy of the Elliott-Yafet parameter in nonmagnetic metals. For this purpose we employ density-functional theory, which has been successfully applied in the past to calculate the spin-mixing parameter in various metals $[9,10]$. Our main finding is that in noncubic transition metals, or generally metallic systems of lowered symmetry, e.g., in the hcp structure, the anisotropy of the Elliott-Yafet parameter can be gigantic. We also demonstrate that in metals with cubic symmetry, such as, e.g., bcc tungsten or fcc gold, this anisotropy is much smaller, although it can still reach large values. Moreover, we analyze the Fermi-surface properties of the spin-mixing anisotropy and provide simple arguments for a microscopic understanding of our results. Our choice of materials ( $5 d$ metals) is based on their similar SOC strength but different crystal structures, which at the end brings about anisotropy values differing by orders of magnitude. 
The Elliott-Yafet theory is based on the observation that the SOC of the lattice ions causes the Bloch eigenstates to be a superposition of spin-up and spin-down character. This superposition is often called spin mixing. The spinorbit Hamiltonian can be divided into a spin-conserving $\xi(L S)_{\|}$and a spin-flip part $\xi(L S)^{\uparrow}$, given respectively by the first and second parts of the right-hand side of the following expression:

$$
\xi \mathbf{L} \cdot \mathbf{S}=\xi L_{\hat{\mathbf{s}}} S_{\hat{\mathbf{s}}}+\frac{1}{2} \xi\left(L_{\hat{\mathbf{s}}}^{+} S_{\hat{\mathbf{s}}}^{-}+L_{\hat{\mathbf{s}}}^{-} S_{\hat{\mathbf{s}}}^{+}\right) .
$$

Here, $\xi$ is the SOC strength, $\hat{\mathbf{s}}$ is a unit vector in the direction of the spin- (and generally angular-momentum-) quantization axis (defining the SQA), $\mathbf{L}$ and $\mathbf{S}=\frac{\hbar}{2} \boldsymbol{\sigma}$ are the orbital and spin angular-momentum operators, respectively, $L_{\hat{\mathbf{s}}}=$ $\mathbf{L} \cdot \hat{\mathbf{s}}, S_{\hat{\mathbf{s}}}=\mathbf{S} \cdot \hat{\mathbf{s}}, L_{\hat{\mathbf{s}}}^{ \pm}$and $S_{\hat{\mathbf{s}}}^{ \pm}$are the corresponding raising and lowering operators for orbital and spin angular momentum in the reference frame specified by the direction vector $\hat{\mathbf{s}}$, and $\boldsymbol{\sigma}$ is the vector of Pauli matrices. It is clear that the dot product $\mathbf{L} \cdot \mathbf{S}$ is independent of $\hat{\mathbf{s}}$, leaving the eigenenergies of the Hamiltonian invariant with respect to the SQA. However, the spin-conserving and spin-flip parts separately depend on the choice of the SQA.

The time-reversal and space-inversion symmetries imply that the eigenenergies of the system at any Bloch momentum $\mathbf{k}$ are at least twofold degenerate, with the corresponding states taking the form [2]

$$
\begin{aligned}
& \Psi_{\mathbf{k} \hat{\mathbf{s}}}^{+}(\mathbf{r})=\left[a_{\mathbf{k} \hat{\mathbf{s}}}(\mathbf{r})|\uparrow\rangle_{\hat{\mathbf{s}}}+b_{\mathbf{k} \hat{\mathbf{s}}}(\mathbf{r})|\downarrow\rangle_{\hat{\mathbf{s}}}\right] e^{i \mathbf{k} \cdot \mathbf{r}}, \\
& \Psi_{\mathbf{k} \hat{\mathbf{s}}}^{-}(\mathbf{r})=\left[a_{-\mathbf{k} \hat{\mathbf{s}}}^{*}(\mathbf{r})|\downarrow\rangle_{\hat{\mathbf{s}}}-b_{-\mathbf{k} \hat{\mathbf{s}}}^{*}(\mathbf{r})|\uparrow\rangle_{\hat{\mathbf{s}}}\right] e^{i \mathbf{k} \cdot \mathbf{r}} .
\end{aligned}
$$

The two spin states $|\uparrow\rangle_{\hat{\mathbf{s}}}$ and $|\downarrow\rangle_{\hat{\mathbf{s}}}$ are eigenstates of $\mathbf{S} \cdot \hat{\mathbf{s}}$, e.g., if $\hat{\mathbf{s}} \| z,|\uparrow\rangle_{z}$ and $|\downarrow\rangle_{z}$ are the eigenstates of the $S_{z}$ operator. The functions $a_{\mathbf{k} \hat{\mathbf{s}}}(\mathbf{r})$ and $b_{\mathbf{k} \hat{\mathbf{s}}}(\mathbf{r})$ exhibit the periodicity of the lattice. We define $b_{\mathbf{k} \hat{\mathbf{s}}}^{2}$ as the unit cell integral $\int d^{3} r\left|b_{\mathbf{k} \hat{\mathbf{s}}}(\mathbf{r})\right|^{2}$.

For fixed direction $\hat{\mathbf{s}}$, the degenerate $\Psi_{\mathbf{k} \hat{\mathbf{s}}}^{+}$and $\Psi_{\mathbf{k} \hat{\mathbf{s}}}^{-}$states [and the corresponding $a_{\mathbf{k} \hat{\mathbf{s}}}(\mathbf{r})$ and $\left.b_{\mathbf{k} \hat{\mathbf{s}}}(\mathbf{r})\right]$ can be chosen, by linear combination, such that the spin-expectation value $\left\langle S_{\hat{\mathbf{s}}}\right\rangle_{\mathbf{k}}=\left\langle\Psi_{\mathbf{k} \hat{\mathbf{s}}}^{+}\left|S_{\hat{\mathbf{s}}}\right| \Psi_{\mathbf{k} \hat{\mathbf{s}}}^{+}\right\rangle=-\left\langle\Psi_{\mathbf{k} \hat{\mathbf{s}}}^{-}\left|S_{\hat{\mathbf{s}}}\right| \Psi_{\mathbf{k} \hat{\mathbf{s}}}^{-}\right\rangle$is maximal. The spin-mixing parameter is then given by $b_{\mathbf{k} \hat{\mathbf{s}}}^{2}=1 / 2-$ $\left\langle S_{\hat{\mathbf{s}}}\right\rangle_{\mathbf{k}} / \hbar$ and is usually small, due to the weakness of the SOC. In this case the Bloch states are of nearly pure spin character. However, at special spin-flip hot spots in the Brillouin zone (BZ), e.g., accidental degeneracies, BZ boundaries, or other high symmetry points $[11,12], b_{\mathbf{k} \hat{\mathbf{s}}}^{2}$ may increase significantly up to $\frac{1}{2}$, which corresponds to the case of fully spin-mixed states. The Fermi-surface (FS) averaged spin-mixing, or Elliott-Yafet, parameter is given by

$$
b_{\hat{\mathbf{s}}}^{2}=\frac{1}{n\left(E_{F}\right)} \frac{1}{\hbar V_{\mathrm{BZ}}} \int_{\mathrm{FS}} \frac{b_{\mathbf{k} \hat{\mathbf{s}}}^{2}}{|\mathbf{v}(\mathbf{k})|} d^{2} k,
$$

where $\mathbf{v}(\mathbf{k})$ is the Fermi velocity and $V_{\mathrm{BZ}}$ is the BZ volume. The normalization by the density of states at the Fermi level, $n\left(E_{F}\right)=1 /\left(\hbar V_{\mathrm{BZ}}\right) \int_{\mathrm{FS}}|\mathbf{v}(\mathbf{k})|^{-1} d^{2} k$, ensures that $0 \leq b_{\hat{\mathbf{s}}}^{2} \leq \frac{1}{2}$.
Our calculations are based on the local density approximation to density-functional theory [13], employing the Korringa-Kohn-Rostoker Green-function method [14] in the atomic sphere approximation and solving the Dirac equation with an angular-momentum cutoff of $\ell_{\max }=4$. We choose a grid of at least $200 \mathbf{k}$ points along each direction in the full $\mathrm{BZ}$, resulting in about $10^{7}$ points at the FS. We follow the procedure described in Ref. [15] to maximize the spin component $\left\langle S_{\hat{\mathbf{s}}}\right\rangle_{\mathbf{k}}$ at the FS points. The details of determination of the Fermi surface and corresponding FS integration will be published elsewhere.

First we discuss in detail hep osmium, which exhibits a uniaxial crystal structure. The Fermi surface of Os, presented in Figs. 1(a) and 1(b), consists of two nested sheets, a surrounding surface crossing the BZ boundary, and little hole pockets " $P$." Analyzing the distribution of the spin-mixing parameter $b_{\mathbf{k} \hat{\mathbf{s}}}^{2}$ on the FS, we observe a strong dependence on the SQA, evident from comparing Figs. 1(a) and 1(b). For $\hat{\mathbf{s}}$ along the $c$ axis of the crystal [Fig. 1(a)], the spin mixing is relatively uniform $\left(b_{\mathbf{k} \hat{\mathbf{s}}}^{2} \approx\right.$ 0.05 ) for large areas of the FS, reaching larger values near the pockets. However, this picture changes drastically when $\hat{\mathbf{s}}$ is parallel to the $a b$ plane [Fig. 1(b)]. In this case, areas with full spin mixing (red, $b_{\mathbf{k} \hat{\mathbf{s}}}^{2} \approx 0.5$ ) are prominent, most clearly visible at the caps of the two nested FS sheets (indicated by " $H$ "). Additionally, large areas with smaller, but still strong spin mixing $\left(b_{\mathbf{k} \hat{\mathbf{s}}}^{2} \approx 0.3\right)$ are visible, e.g., in the area denoted by " $B$." Overall, for the two considered cases there is a strong qualitative difference in the $\mathbf{k}$-dependent spin-mixing parameter $b_{\mathbf{k} \hat{\mathbf{s}}}^{2}$.

As for the Fermi-surface averaged $b_{\hat{\mathrm{s}}}^{2}$, we find values of $4.85 \times 10^{-2}$ and $7.69 \times 10^{-2}$ for $\hat{\mathbf{s}}$ along the $c$ axis and parallel to the $a b$ plane, respectively, yielding thus a gigantic anisotropy of the Elliott-Yafet parameter, defined as $\mathcal{A}=\left[\max _{\hat{\mathbf{s}}}\left(b_{\hat{\mathbf{s}}}^{2}\right)-\min _{\hat{\mathbf{s}}}\left(b_{\hat{\mathbf{s}}}^{2}\right)\right] / \min _{\hat{\mathbf{s}}}\left(b_{\hat{\mathbf{s}}}^{2}\right)$, of $59 \%$. The anisotropy with respect to rotations of the SQA within the $a b$ plane is, on the other hand, negligible. These two limiting cases are contained in Fig. $1(\mathrm{~g})$, in which the value of $b_{\hat{\mathbf{s}}}^{2}$ is shown as a function of all possible directions of $\hat{\mathbf{s}}$ on the unit sphere. The absent (or very small) anisotropy within the $a b$ plane is reflected in the rotationally invariant color scale around the $c$ axis, as opposed to the large difference between the $a b$ plane and the $c$ axis. A detailed analysis reveals that the largest contribution to $\mathcal{A}$ comes from the somewhat extended spin-flip hot areas with high or intermediate values of $b_{\mathbf{k} \hat{\mathbf{s}}}^{2}>0.15$.

We delve into the anisotropy of the spin mixing at the area around $H$ by means of a numerical experiment. Analyzing the band structure of Os along the high symmetry line from the center of the $\mathrm{BZ}$ ( $\Gamma$ point) through the $H$ point to the center of the hexagonal face ( $A$ point), in Fig. 1(e), we see two bands crossing the Fermi level at $H$, representing the two nested FS sheets of Figs. 1(a) and $1(\mathrm{~b})$. The splitting $\Delta_{\text {SOC }}$ between these two bands is due to SOC, as we have verified by the fact that they fall on 


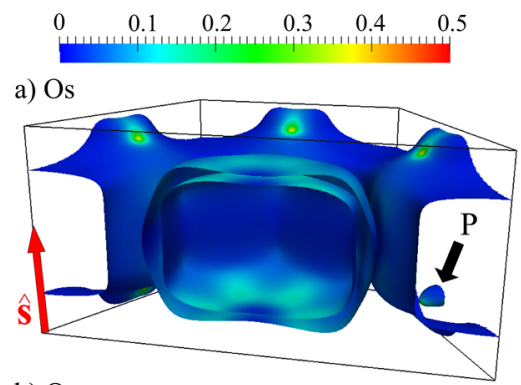

b) $\mathrm{Os}$

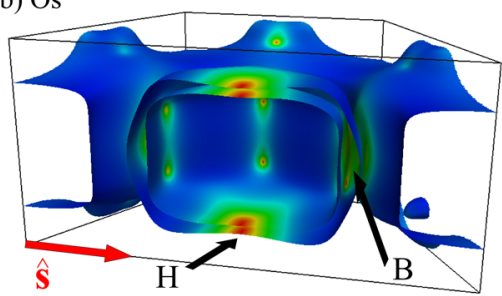

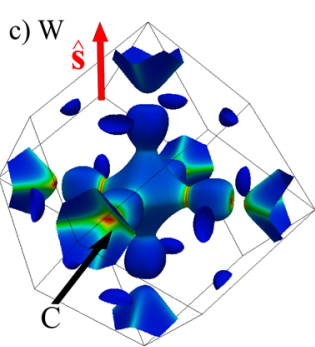

d) $\mathrm{W}$

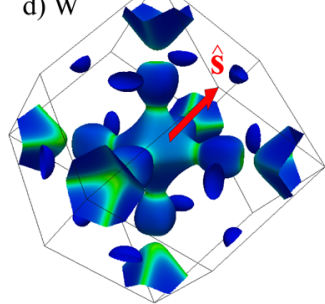

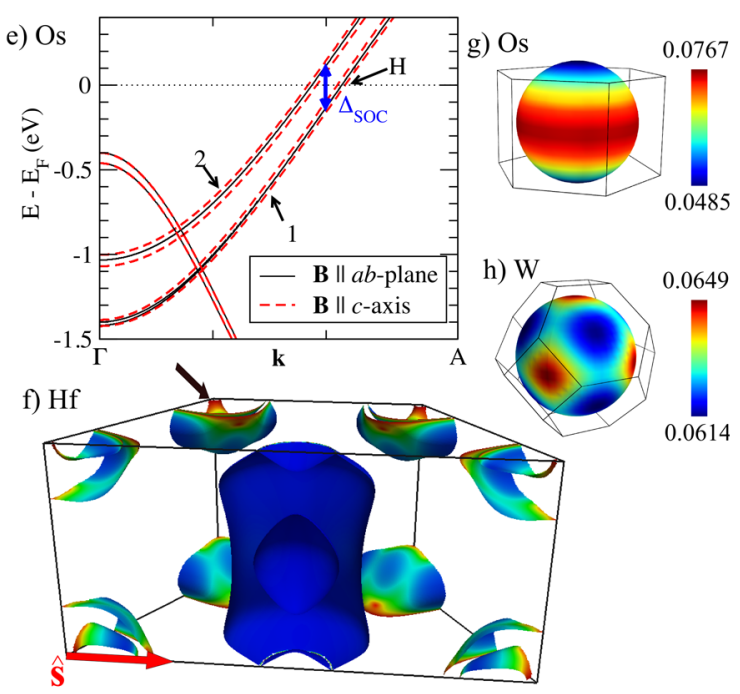

FIG. 1 (color online). Fermi surfaces of Os (a), (b), W (c), (d), and Hf (f). For an illustration of the nested sheets of Os and Hf, only half of the Fermi surface is shown. The spin-mixing $b_{\mathbf{k} \hat{\mathbf{s}}}^{2}$ is shown in terms of a color code with the SQA $\hat{\mathbf{s}}$ (red arrows at the lower left corners) along the $c$ axis (a) and the $a b$ plane (b). The splitting introduced by SOC and by a Zeeman-like field is shown in the band structure of Os (e) along the $\Gamma-A$ direction (BZ center to hexagonal-face center). For W, the cases $\hat{\mathbf{s}} \|$ [001] and [111] are shown in (c) and (d), respectively. In (g) and (h), the Elliott-Yafet parameter $b_{\hat{\mathrm{s}}}^{2}$ is shown as a function of the SQA direction for Os and W, respectively [in $(\mathrm{g})$ and $(\mathrm{h})$ the color scale is different than in the Fermi-surface plots]. The average of $b_{\hat{\mathbf{s}}}^{2}$ over all directions of $\hat{\mathbf{s}}$, corresponding to polycrystalline samples, are 0.0666 for Os and 0.0627 for W. (f) Hf Fermi surface with $\hat{\mathbf{s}}$ parallel to the $a b$ plane. An arrow indicates one of the spin-flip hot loops, clearly visible in red on the hexagonal face of the BZ. The hot loops vanish when $\hat{\mathbf{s}}$ is rotated to the $c$ axis, resulting in an anisotropy of $830 \%$ of the Elliott-Yafet parameter.

top of each other when the SOC strength is scaled down (not shown). Each band is twofold degenerate due to timereversal and inversion symmetry. This remanent degeneracy can be lifted by applying a small Zeeman-like field $\mathbf{B}$ coupling to the Bloch states via a term $\boldsymbol{\sigma} \cdot \mathbf{B}$, which breaks the time-reversal symmetry and sets the SQA in the direction of $\mathbf{B}$. We choose a weak $\mathbf{B}$ field with a magnitude of $40 \mathrm{meV}$ and vary its direction. In Fig. 1(e), we clearly observe a splitting of bands " 1 " and " 2 " for $\mathbf{B}$ along the $c$ axis (dashed red lines). However, for $\mathbf{B}$ in the $a b$ plane, the degenerate pairs 1 and 2 do not split (solid black lines). We can relate this result to our findings for $b_{\mathbf{k} \hat{\mathbf{s}}}^{2}$ by employing perturbation theory arguments: In first order, the energy shift of a state in the presence of a small $\mathbf{B}$ field is proportional to the state's spin-expectation value. This numerical experiment shows that in the case that $\mathbf{B} \| a b$ plane the states are fully spin mixed, just as found by the calculation of $b_{\mathbf{k} ; \hat{s} \| a b}^{2}$. It also reveals an anisotropy of the susceptibility that follows from the anisotropy of the spinmixing parameter.

We are now in a position to give a simple line of arguments demonstrating the microscopic mechanism that leads to a large anisotropy in general. As a working example, we use the calculated giant anisotropy at and in the vicinity of the $H$ point in Os. Important in the setup is the presence of a degeneracy or near degeneracy at $E_{F}$, here at the point $H$, of Bloch wave functions $\Psi_{\mathbf{k}}^{1,2}$, which in a tightbinding picture we represent as $\Psi_{\mathbf{k}}^{1,2}=\sum_{l m} c_{\mathbf{k} l m}^{1,2}|l, m\rangle$. Here, $|l, m\rangle$ are eigenstates of $\mathbf{L}_{\hat{\mathbf{s}}}$ for a given SQA $\hat{\mathbf{s}}$ in the crystal, e.g., the $z$ axis. The requirement for a large anisotropy is met if the matrix elements of the spin-flip SOC operator $\xi(L S)^{\uparrow \downarrow}$ between $\Psi_{\mathbf{k}}^{1}$ and $\Psi_{\mathbf{k}}^{2}$ vanish. This occurs if the expansions above exclude terms with $l_{1}=l_{2}$ and $\left|m_{1}-m_{2}\right|=1$, where $\left|l_{1}, m_{1}\right\rangle$ contributes to $\Psi_{\mathbf{k}}^{1}$ and $\left|l_{2}, m_{2}\right\rangle$ to $\Psi_{\mathbf{k}}^{2}$. Here, concretely, we have found that only the orbitals $|d,-1\rangle$ and $|d,+1\rangle$ contribute to the bands 1 and 2 at $H$. These are superimposed to form the oriented orbitals $d_{x z}$ and $d_{y z}$ which are the actual eigenstates of the crystal field. Then the lifting of degeneracy is due only to the spin-conserving SOC $\xi(L S)_{\|}$which causes no spin mixing.

Suppose now that the SQA (and together with it the axis for quantization of orbital angular momentum) is rotated around $y$, from $z$ to $\bar{z}=x$. In the new frame $(\bar{x} \bar{y} \bar{z})$ we denote the orbital functions and angular-momentum indices with an overline. The oriented orbitals have a new resolution with respect to the new axes. For example, $d_{y z}$ becomes $d_{\bar{x} \bar{y}}$ having a projection on $|\overline{d, \pm 2}\rangle$, while $d_{x z}$ becomes $d_{\bar{x} \bar{z}}$ having a projection on $|\overline{d, \pm 1}\rangle$. As a result, the expansions of $\Psi_{\mathbf{k}}^{1,2}$ with respect to the new frame include orbitals with $l_{1}=l_{2}$ and $\left|\bar{m}_{1}-\bar{m}_{2}\right|=1$ at the same energy, allowing for nonzero matrix elements of the spin-flip part of the SOC. For the SQA along $z$, the system is "protected" against large-amplitude spin-flip transitions, while for the SQA along $x$ or $y$ spin-flip transitions are favored. What we have demonstrated here is that the matrix elements of the spin-conserving and spin-flip part of 
the SOC can depend so strongly on the SQA that the spin flip vanishes in one direction while it is maximal in another. For example, while in the original frame the SOCinduced band splitting at $H$ arises from $\xi(L S)_{\|}$, in the new frame the exact same splitting arises from $\xi(L S)^{\uparrow}$, leading to a spin mixing of $\frac{1}{2}$. It is, therefore, the direction of the SQA that dictates which part of the Hamiltonian causes most of the splitting, even though the sum of the two contributions is independent of the direction of the SQA. Other states, lying far away in energy due to the crystal field splitting, play only a small role in the final result.

The mechanism for large anisotropy of the spin-mixing parameter described above is of course not only specific to the $d$ states of Os, but it is also responsible for gigantic values of $\mathcal{A}$ that we find for hcp Lu (200\%), hcp $\operatorname{Re}(88 \%)$, and hcp Hf (830\%). Particularly in hcp metals, there is a special symmetry at the hexagonal face of the BZ that is lifted only by the SOC [16]. Thus, whenever the FS of an hcp metal happens to cut through the hexagonal face, the resulting contour can obtain full spin mixing depending on the SQA, as shown in Fig. 1(f) for Hf. These looplike contours, or spin-flip hot loops, are a source of extremely high anisotropy. The Fermi surfaces of $\mathrm{Lu}, \mathrm{Re}$, and Hf, for example, contain such loops, but the one of Os does not, since it does not cut through the hexagonal face. We further observe that the magnitude of the effect can be strongly enhanced by the large extension of the two near-degenerate, parallel sheets of the FS, resulting in a spin-flip hot area around the point of near degeneracy. For example, in Os we obtain a hot area around point $H$ instead of a single hot spot at $H$, while the position and topology of such areas generally depend on the FS and electronic structure.

We now turn to tungsten, which has a bcc lattice structure. When $\hat{\mathbf{s}} \|[001], b_{\mathbf{k} \hat{\mathbf{s}}}^{2}$ exhibits hot spots in directions perpendicular to $\hat{\mathbf{s}}$ (denoted by " $C$ ") [Fig. 1(c)] but not at the rotationally equivalent points along the $z$ axis, following the formation scenario similar to that at the $H$ point in Os. Additionally, many states with smaller spin mixing $\left(0.2<b_{\mathbf{k} \hat{\mathbf{s}}}^{2}<0.3\right)$ are present at the FS, leading to $b_{\hat{\mathbf{s}}}^{2}=$ $6.49 \times 10^{-2}$. For SQA along another high symmetry direction, $\hat{\mathbf{s}} \|[111]$ in Fig. 1(d), the intensity at the point $C$ is reduced, but a large area with smaller spin mixing is clearly present, resulting in $b_{\hat{\mathrm{s}}}^{2}=6.14 \times 10^{-2}$. For SQA along [110], we find $b_{\hat{\mathrm{s}}}^{2}=6.26 \times 10^{-2}$. This leads to an anisotropy $\mathcal{A}=6 \%$, which is still large but 1 order of magnitude smaller than in hep Os. In fact, we also predict rather small anisotropy in other cubic transition metals, such as Ir $(1 \%)$ and Pt $(0.4 \%)$. This observation is similar to the dependence of the magnetocrystalline anisotropy energy and anisotropy of the anomalous Hall effect $[17,18]$ on the symmetry of the lattice in ferromagnets: For the hop structure with lower symmetry SOC contributes to $\mathcal{A}$ in second order, while it enters only in fourth order in cubic crystals. Generally, we expect the integrated value $b_{\hat{s}}^{2}$ to exhibit the full point-group symmetry of the lattice [evident in
Figs. 1(g) for Os and (h) for W], even if the map of $b_{\mathbf{k} \hat{\mathbf{s}}}^{2}$ has a lower symmetry. The comparatively large anisotropy value in $\mathrm{W}$ is partly a consequence of the $d$ states, which yield a strong directional anisotropy of the Fermi surface. In contrast to this, the FS of fcc gold consists of $s$-like states and can be regarded as almost spherical. For the Elliott-Yafet parameter in $\mathrm{Au}$, we find $b_{\hat{\mathrm{s}}}^{2} \approx 3.25 \times 10^{-2}$, i.e., the same order of magnitude as in $\mathrm{W}$ and Os, but the anisotropy is only $0.1 \%$.

In conclusion, spin relaxation in metals can strongly depend on the orientation of the injected-electron spin axis due to a corresponding anisotropy of the ElliottYafet parameter. The anisotropy is expected to be largest in noncubic crystals and in the presence of extended nested Fermi-surface sheets that are almost degenerate, resulting in extended spin hot areas or hot loops instead of singular spin hot spots; especially critical are cases where the splitting between nested sheets is caused primarily by the spin-orbit coupling. Since there is no theoretical limit on the area of the nested sheets in this scenario, the anisotropy can in principle exceed the large values calculated here and is an effect worth investigating on a number of metals and ordered alloys. While the precise scattering mechanism will naturally affect the anisotropy, our results should be measurable even if the exact value can deviate somewhat from the one of the Elliott approximation. In this respect it seems most beneficial to investigate the temperature dependence of the effect, which can be attributed mainly to phonons. We furthermore expect that anisotropy effects should also be present in metallic alloys, heterostructures, or ultrathin films, which we leave for future work.

We are indebted to J. Fabian for an introduction to the field and for discussions. We are also indebted to R. Zeller and P.H. Dederichs for their invaluable help in the Korringa-Kohn-Rostoker formalism and to G. Bihlmayer, D. V. Fedorov, and P. Zahn for discussions. We acknowledge funding under DFG Projects No. MO 1731/3-1 and No. SPP-1538 "Spin Caloric Transport," the HGF YIG Program VH-NG-513, as well as computing time at the Jülich Supercomputing Centre.

*Ph.Mavropoulos@fz-juelich.de

[1] I. Žutić, J. Fabian, and S. D. Sarma, Rev. Mod. Phys. 76, 323 (2004).

[2] R. J. Elliott, Phys. Rev. 96, 266 (1954).

[3] Y. Yafet, in Solid State Physics, edited by F. Seitz and D. Turnbull (Academic, New York, 1963), Vol. 14, p. 1.

[4] An anisotropy of spin relaxation has been measured in supported graphene layers [5] and discussed for semiconductors [6]; however, these are systems without space-inversion symmetry where the Dyakonov-Perel mechanism is dominant.

[5] N. Tombros, S. Tanabe, A. Veligura, C. Jozsa, M. Popinciuc, H. T. Jonkman, and B.J. van Wees, Phys. Rev. Lett. 101, 046601 (2008). 
[6] N. S. Averkiev and L. E. Golub, Semicond. Sci. Technol. 23, 114002 (2008).

[7] H. Katayama-Yoshida, T. Fukushima, V. A. Dinh, and K. Sato, Jpn. J. Appl. Phys. 46, L777 (2007); A. Sugihara, M. Kodzuka, K. Yakushiji, H. Kubota, S. Yuasa, A. Yamamoto, K. Ando, K. Takanashi, T. Ohkubo, and K. Hono, Appl. Phys. Express 3, 065204 (2010).

[8] F. Freimuth, S. Blügel, and Y. Mokrousov, Phys. Rev. Lett. 105, 246602 (2010).

[9] M. Gradhand, M. Czerner, D. V. Fedorov, P. Zahn, B. Y. Yavorsky, L. Szunyogh, and I. Mertig, Phys. Rev. B 80, 224413 (2009).

[10] D. Steiauf and M. Fähnle, Phys. Rev. B 79, 140401 (2009); K. Carva, M. Battiato, and P. M. Oppeneer, Phys. Rev. Lett. 107, 207201 (2011).
[11] J. Fabian and S.D. Sarma, Phys. Rev. Lett. 81, 5624 (1998).

[12] J. Fabian and S.D. Sarma, Phys. Rev. Lett. 83, 1211 (1999).

[13] S. H. Vosko, L. Wilk, and M. Nusair, Can. J. Phys. 58, 1200 (1980).

[14] H. Ebert and R. Zeller, The SPR-TB-KKR Package, http:// olymp.cup.uni-muenchen.de/ak/ebert/SPR-TB-KKR.

[15] S. Heers, Ph.D. thesis, RWTH Aachen, 2011, http:// darwin.bth.rwth-aachen.de/opus3/volltexte/2011/3827.

[16] N. W. Ashcroft and D. N. Mermin, Solid State Physics (Saunders, Philadelphia, 1976), p. 299.

[17] C. Zener, Phys. Rev. 96, 1335 (1954).

[18] E. Roman, Y. Mokrousov, and I. Souza, Phys. Rev. Lett. 103, 097203 (2009). 